\title{
XII. A preliminary note on the relation between primary and secondary Röntgen radiation
}

\section{H.S. Allen M.A. B.Sc.}

To cite this article: H.S. Allen M.A. B.Sc. (1902) XII. A preliminary note on the relation between primary and secondary Röntgen radiation, Philosophical Magazine Series 6, 3:13, 126-128, DOI: $10.1080 / 14786440209462745$

To link to this article: http://dx.doi.org/10.1080/14786440209462745

册 Published online: 09 Jun 2010.

Submit your article to this journal $\sqsubset \pi$

Џll Article views: 3

Q View related articles $₫$ 
because of the periodic doubling of certain lines in their spectra. Such doubling would occur owing to the wellknown Doppler effect, when the two components are moving one away from, and the other towards, the earth. The orbital velocity ean be computed from the separation of the lines, and amounts in some cases to as much as 150 miles per second or many times that of the earth in its orbit. Now consider the moment when the two components are in the line joining the star to the earth. One A will be moving to the right, and the other $B$ to the left with a velocity of 150 miles per second. If the effect of motion of the source on aberration is at all comparable with that of motion of the receiver, we should expect (when the light then leaving the star reaches the earth) to see star A displaced to the right and $B$ to the left. The star would appear double in a telescope, and the apparent doubling would occur periodically, and alternately with the separation of the spectroscopic lines. That no such doubling is seen is very strong, if not absolutely conclusive evidence of the fact we set out to establish, that motion of the source does not affect aberration and it forms a real logical basis for postulating an æther.

XII. A Preliminary Note on the Relation between Prinary and Secondary Röntgen Radiation. By H. S. Allen, M.A., B.Sc.*

W HEN the Röntgen radiation from a focus-tube falls upon the surface of certain solids, the surface becomes the source of a secondary radiation. These "Secondary Röntgen Rays" are in many respects similar to the ordinary rays which produce them. Their properties have been investigated by Sagnac $\dagger$, Perrin $\ddagger$, and Townsend $\S$. Both primary and secondary rays render a gas throngh which they pass a conductor, but while the primary rays suffer very little absorption the secondary rays are almost entirely absorbed within a few millimetres at atmospheric pressure. The object of the present investigation is to compare the total ionization produced by the secondary rays with that which would be produced by the primary if they continued their path in the original medium. Then, supposing that the total ionization, if not equal to, is at least proportional to the

* Communicated by Prof. J. J. Thomson.

+ Journal de Physique, 3rd series, viii. Feb. 1899.

$\ddagger$ Comptes Rendus, cxxiv. p. 455.

\$ Proc. Camb. Phil. Soc. X. Part iv. 1900. 
energy of the radiation, we shall obtain a measure of the ratio of the energies in the two kinds of radiation.

The experimental work involves two separate determinations. In the first place, the ratio of the total ionization caused by the secondary rays to that produced within a known space by the primary; and, secondly, the absorption experienced by the primary rays in traversing a known distance.

With the assumption just stated, the absorption of energy in a parallel beam of rays in traversing a thin layer of gas of thickness $d l$ at a point where the intensity is $\bar{I}$ is equal to $\lambda \mathrm{I} d l$, where $\lambda$ is the coefficient of absorption of the gas.

For simplicity we may consider the radiation contained in a cone of small solid angle $d \omega$, with its vertex at the anticathode. If I denote the intensity of radiation at a distance $r$ from the vertex, we obtain the following differential equation for determining $I$ in terms of $r$ :

$$
\begin{gathered}
\left(\mathrm{I}+\frac{d \mathrm{I}}{d r} d r\right)(r+d r)^{2} d \omega-\mathrm{I} r^{2} d \omega=\lambda \mathrm{I} r^{2} d r d \omega, \\
\therefore \frac{d}{d r}\left(\mathrm{I} r^{2}\right)=\lambda \mathrm{I} r^{2} .
\end{gathered}
$$

The solution of this equation is

$$
\mathrm{I} r^{2}=\mathrm{I}_{0} r_{0}{ }^{2} e^{-\lambda\left(r-r_{0}\right)} .
$$

The ionization produced by the rays in the element of volume may be expressed as $k I r^{2} d r d \omega$, where $k$ is a constant.

Hence the total ionization produced within the boundary of the cone between $r_{0}$ and infinity is

$$
\int_{r_{0}}^{\infty} k \mathrm{I} r^{2} d r d \omega=\frac{k \mathrm{I}_{0} r_{0}^{2} d \omega}{\lambda}
$$

The ionization actually measured is that produced in the same cone between $r_{0}$ and $r_{0}+d r_{0}$, and, using the same notation, may be written

$$
k \mathrm{I}_{0} r_{0}{ }^{2} d r_{0} d \omega .
$$

Thus the total ionization may be obtained by dividing this, the observed quantity, by $\lambda d r_{0}$.

The method employed in the first determination, that of the ionization produced by the secondary rays, was similar to that used by Perrin (loc. cit.), and consisted in the comparison of the rates of leak from two condensers, the leak in 
the first being due to primary radiation alone, that in the second to primary and secondary radiation.

The method employed in measuring the absorption of the primary rays was also a comparative one, and was practically identical with that of Rutherford *.

As yet the determinations have only been completed in the case of a single gas - sulphuretted hydrogen-in which the coefficient of absorption is comparatively large. In this gas, calling the ionization produced by the primary rays in a distance of $2.5 \mathrm{~cm}$. 100 , the ionization caused by the secondary rays from a freshly polished brass plate, $10 \mathrm{~cm}$. by $5 \mathrm{~cm}$. in area, amounted to 11 . The variation due to the absorption of $20 \mathrm{~cm}$. of sulphuretted hydrogen was found to be 3.9 per cent., giving as the value of $\lambda 1.9 \times 10^{-3}$.

Hence the ratio of the total ionization caused by the secondary radiation from a brass plate to the total ionization for the primary radiation is equal to

$$
\frac{11}{100} \times 2 \cdot 5 \times 1 \cdot 9 \times 10^{-3} \text {, }
$$

or $\frac{1}{1900}$ very nearly.

From this it follows on the foregoing assumptions that the total energy of the secondary radiation only amounts to about one two-thousandth part of the energy of the primary radiation.

The work here described was carried out at the Cavendish Laboratory at the suggestion of Professor J. J. Thomson, to whom I am glad to express my indebtedness.

Trinity College, Cambridge.

XIII. The Anomalous Dispersion of Sodium Vapour. By R. W. Wood, Professor of Physics in the University of Wisconsint.

$$
\text { [Plates II.-IV.] }
$$

GXPERIMENTAL proof of the dispersion formulæ of E Sellmeier, Helmboltz, and Ketteler has for the most part been based upon observations made upon substances in the solid state or in the state of solution, where the range of absorption extends over a considerable portion of the

* Phil. Mag. [5] xliii. pp. \$59-255 (1897).

+ Read before the Royal Society June 20, 1901. We are indebted to the Royal Society for the use of the stone for the plate and to the Author for defraying part of the expense of reproducing it. 